\title{
First-Principles Molecular Dynamics Insight into the Atomic Level Degradation Pathway of Phosphorene
}

\author{
Jeevesh Kumar and Mayank Shrivastava*
}

Cite This: ACS Omega 2022, 7, 696-704

Read Online

ACCESS | Lلll Metrics \& More | 国 Article Recommendations | st Supporting Information

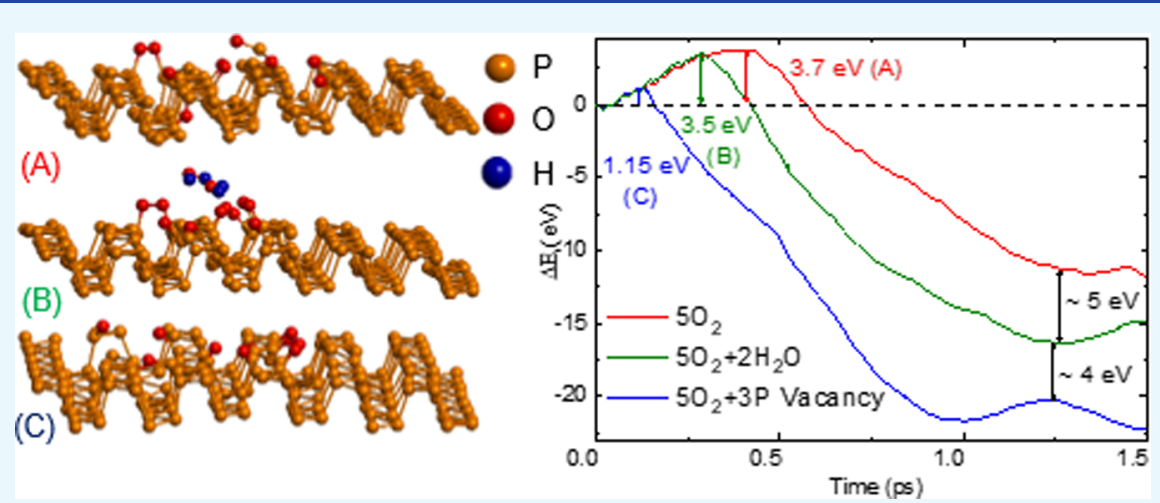

ABSTRACT: Despite its remarkable properties, phosphorene is not promising for device application due to its instability or gradual degradation under ambient conditions. The issue still persists, and no technological solution is available to address this degradation due to a lack of clarity about degradation dynamics at the atomic level. Here, we discuss atomic level degradation dynamics of phosphorene under ambient conditions while investigating the involvement of degrading agents like oxygen and water using density functional theory and first-principles molecular dynamics computations. The study reveals that the oxygen molecule dissociates spontaneously over pristine phosphorene in an ambient environment, resulting in an exothermic reaction, which is boosted further by increasing the partial pressure and temperature. The surface reaction is mainly due to the lone pair electrons of phosphorous atoms, making the degradation directional and spontaneous under oxygen atoms. We also found that while the pristine phosphorene is hydrophobic, it becomes hydrophilic after surface oxidation. Furthermore, water molecules play a vital role in the degradation process by changing the reaction dynamics path of the phosphorene-oxygen interaction and reducing the activation energy and reaction energy due to its catalyzing action. In addition, our study reveals the role of phosphorous vacancies in the degradation, which we found to act as an epicenter for the observed oxidation. The oxygen attacks directly over the vacant site and reacts faster compared to its pristine counterpart. As a result, phosphorene edges resembling extended vacancy are prominent reaction sites that oxidize anisotropically due to different bond angle strains. Our study clears the ambiguities in the kinetics of phosphorene degradation, which will help engineer passivation techniques to make phosphorene devices stable in the ambient environment.

\section{INTRODUCTION}

Phosphorene, an atomically thin sheet of black phosphorous, has all of the potential to fill the gap between graphene and transition-metal dichalcogenides (TMDs) for electronic and optoelectronic applications, mainly due to a wide range of direct band gap, high mobility, and anisotropic electrical and thermal conductivity. ${ }^{1-3}$ However, the material is unsuitable for electronic and optoelectronic applications due to a lack of stability in the ambient environment. ${ }^{4}$ Some of the engineering approaches like encapsulation, ${ }^{5}$ pulsed laser exfoliation, ${ }^{6}$ solvent passivation, ${ }^{7}$ and oxygen plasma etching ${ }^{8}$ have guided to make the material stable. The methods, however, are not very promising for long-term stability, which is a real challenge in terms of material growth, device processing, and packaging for such applications. Understanding the degradation mechanism at the atomic level is a crucial parameter that can help material-level engineering of these issues. Various research groups have explored and explained the role of oxygen, water, and light over the degradation mechanism of phosphorene. ${ }^{9-30}$ The majorly proposed degradation path is the oxidation of phosphorene, followed by etching of the surface oxide by water molecules as investigated by Huang et al., ${ }^{12}$ Druenen et al., ${ }^{14}$ Zhang et al., ${ }^{16}$ Plutnar et al., ${ }^{18}$ and Wang et al. ${ }^{30}$ However, the proposed role of water in degradation kinetics seems

Received: September 27, 2021

Accepted: December 21, 2021

Published: January 1, 2022

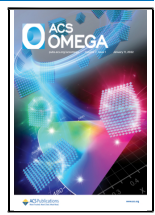




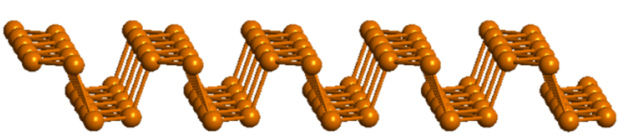

(a)

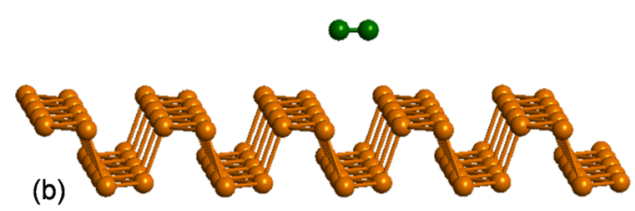

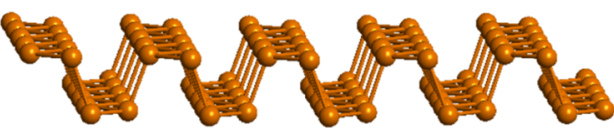

(c)

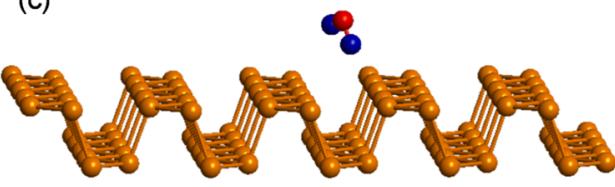

(d)

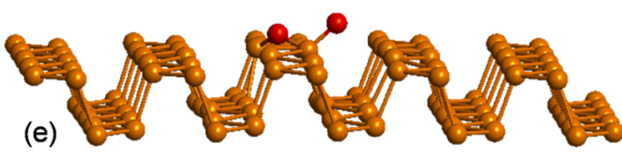

Figure 1. DFT-optimized minimum energy structures of phosphorene with (a) $\mathrm{Ar}$, (b) $\mathrm{N}_{2}$, (c) $\mathrm{CO}_{2}$, (d) $\mathrm{H}_{2} \mathrm{O}$, and (e) $\mathrm{O}_{2}$.
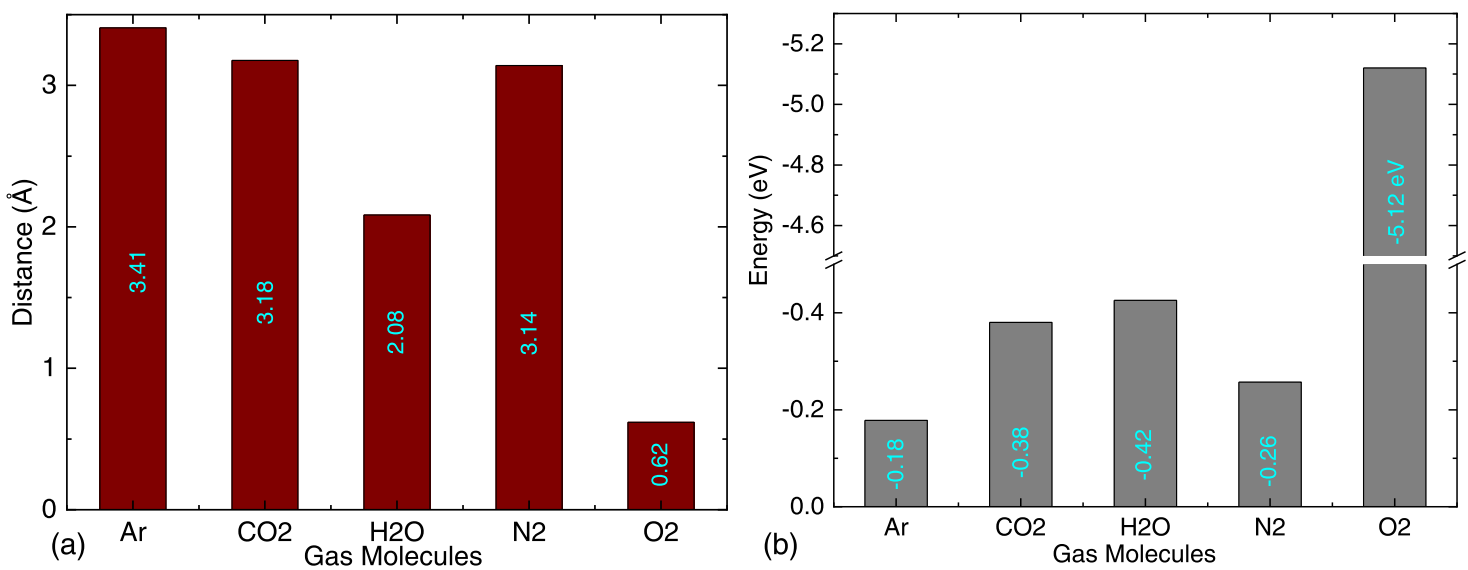

Figure 2. (a) Distance of the molecules from the phosphorene surface after energy optimization. (b) Corresponding adsorption/reaction energy.

ambiguous based on the water-catalyzed degradation study by $\mathrm{Hu}$ et $\mathrm{al}^{26}$ and $\mathrm{pH}$-dependent degradation study by Zhang et $\mathrm{al}^{27}$ Theoretical investigations of the phosphorene degradation mechanism can better picture the degradation dynamics and surface chemistry. Ziletti et al. ${ }^{28}$ have studied phosphorene's possible oxygen orientation and oxidation path using density functional theory (DFT). Eslamibidgoli et al. ${ }^{29}$ have explored the conversion of the phosphorene surface from a hydrophobic to a hydrophilic surface after oxidation based on their molecular dynamics (MD) investigations. However, these studies do not give a complete and clear picture of the degradation kinetics as far as the role of water is concerned. Based on MD and DFT computations, Wang et al. ${ }^{30}$ have predicted that phosphorene degrades via oxidation, followed by surface oxide etching by water. The authors, however, do not incorporate MD investigation of the surface oxidation in the presence of water; thus, they do not show the dynamics of water over the oxidized phosphorene surface. Moreover, due to other parameters like oxygen partial pressure, phosphorous vacancy, temperature, and phosphorene anisotropy, the degradation kinetics is not well understood, demanding extensive computations.

To address these issues, we have investigated the interaction of phosphorene with oxygen and water under different conditions using DFT and first-principles MD simulations. The manuscript starts with the interaction chemistry of phosphorene with different ambient gases, followed by the interaction dynamics of phosphorene with oxygen and the role of the phosphorous lone pair. After that, it explains the behavior of water molecules over pristine as well as oxidized phosphorene. Consequently, the manuscript throws light on the nature of the degradation dynamics in the presence of phosphorous vacancy and anisotropic edge degradations. Finally, it reveals the effect of ambient temperature on the degradation of pristine phosphorene under oxygen.

\section{RESULTS AND DISCUSSION}

Spontaneous degradation of phosphorene is often very fast in an ambient environment. Thus, some major ambient gases, like $\mathrm{N}_{2}, \mathrm{O}_{2}, \mathrm{Ar}, \mathrm{H}_{2} \mathrm{O}$, and $\mathrm{CO}_{2}$, may have a solid chemical affinity toward phosphorene. For investigations, pristine phosphorene was optimized for minimum energy in the presence of all of these ambient gas molecules using DFT (Figure 1).

Ar, $\mathrm{N}_{2}, \mathrm{CO}_{2}$, and $\mathrm{H}_{2} \mathrm{O}$ are adsorbed over phosphorene surfaces without any significant perturbation in their chemical structures (Figure $1 \mathrm{a}-\mathrm{d}$ ), whereas $\mathrm{O}_{2}$ dissociates and renders surface oxides (Figure 1e). Oxygen atoms are in the dangling and bridge positions after bonding with phosphorous. The dangling $\mathrm{P}-\mathrm{O}$ bond length is $1.51 \AA$, while the average bridge $\mathrm{P}-\mathrm{O}$ bond length is $1.66 \AA$. The stable oxygen positions and $\mathrm{P}-\mathrm{O}$ bond lengths are consistent with previous work by Ziletti et al. $^{28}$ Binding energies of all of the molecules with different phosphorene surfaces were calculated using eq 1 , where $E_{\text {sys }}$ is the energy of the optimized phosphorene-molecule system, 


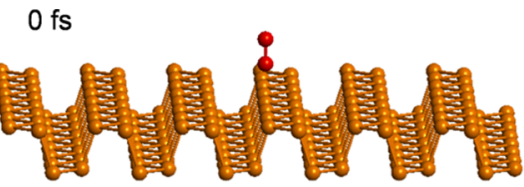

(a)

- 0

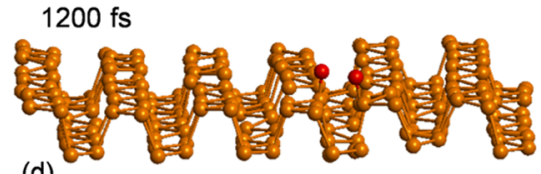

400 fs

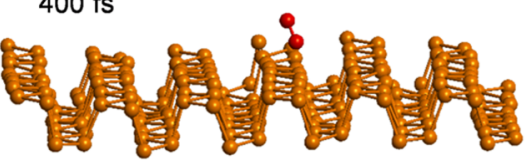

(b)

$1600 \mathrm{fs}$

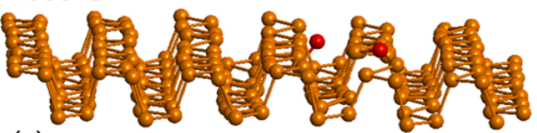

(e)

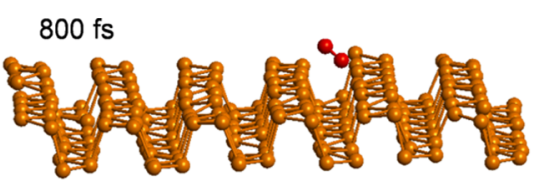

(c)

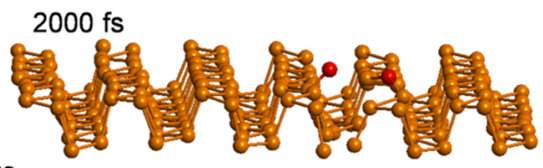

(f)

Figure 3. Status of the $\mathrm{O}_{2}$ molecule over pristine phosphorene in different timestamps, (a) $0 \mathrm{fs},(\mathrm{b}) 400 \mathrm{fs},(\mathrm{c}) 800 \mathrm{fs}$, (d) $1200 \mathrm{fs}$, (e) $1600 \mathrm{fs}$, and (f) $2000 \mathrm{fs}$, during MD progress. Corresponding full dynamics video is available in the Supporting Information (SV1).

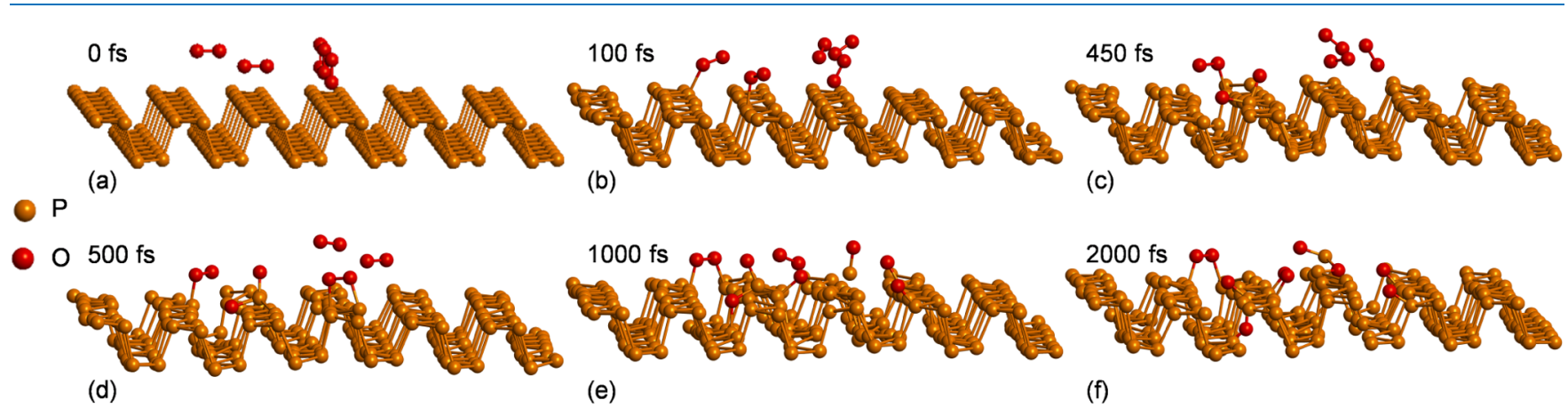

Figure 4. Status of five $\mathrm{O}_{2}$ molecules over pristine phosphorene in different timestamps, (a) $0 \mathrm{fs}$, (b) $100 \mathrm{fs}$, (c) $450 \mathrm{fs}$, (d) $500 \mathrm{fs}$, (e) $1000 \mathrm{fs}$, and (f) $2000 \mathrm{fs}$, during MD progress. Corresponding full dynamics video is available in the Supporting Information (SV2).
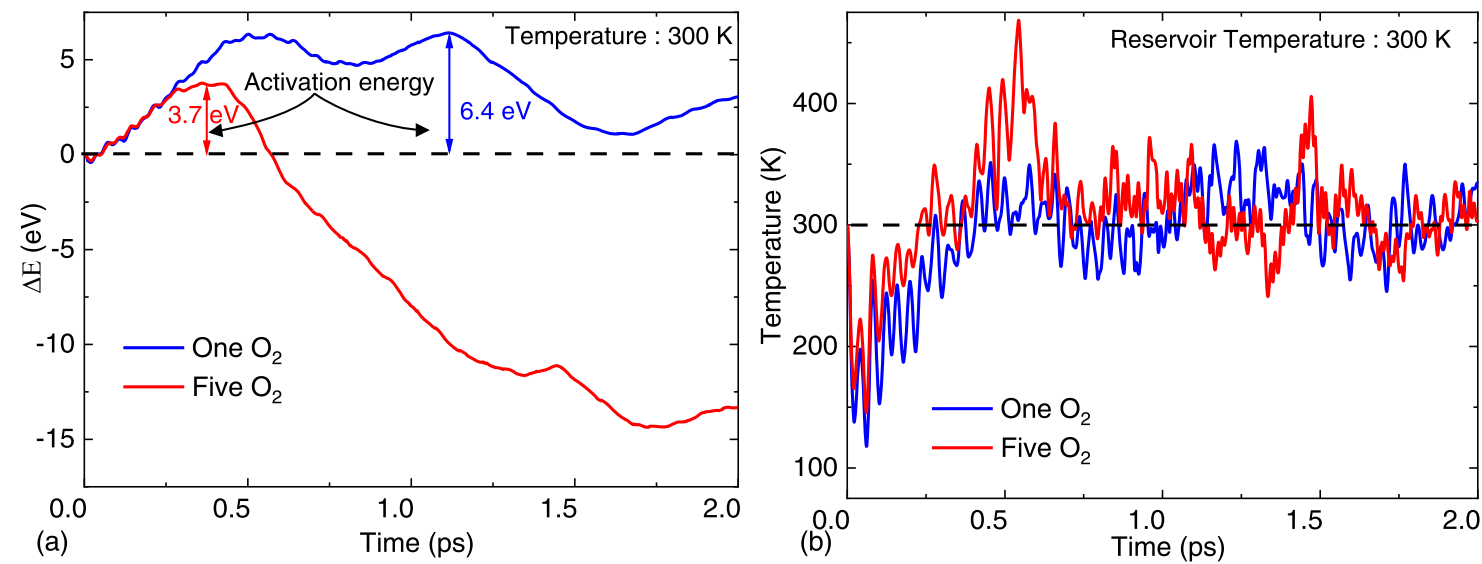

Figure 5. (a) Change in energy and (b) temperature of the phosphorene-oxygen systems with reaction progress in time.

while $E_{\text {molecule }}$ and $E_{\text {Phosphorene }}$ are optimized energies of the corresponding molecule and phosphorene, respectively, in isolated conditions.

$$
\Delta E=E_{\text {sys }}-\left(E_{\text {molecule }}+E_{\text {Phosphorene }}\right)
$$

Bond distance (Figure 2a) and bond energy (Figure 2b), calculated using eq 1 , confirm that other gases are physically adsorbed over the surface. These gases sit more than $2 \AA$ above the phosphorene plane and release less than $0.5 \mathrm{eV}$ energy, which signifies that they are in physical interactions with phosphorene. $\mathrm{H}_{2} \mathrm{O}$ has the most physical affinity to phosphorene than other adsorbed gases due to the possible hydrogen bond between $\mathrm{H}-\mathrm{OH}$ and the lone pair of phosphorous atoms. On the other hand, the interaction of oxygen with phosphorene is highly exothermic $(-5.12 \mathrm{eV})$. The released energy is very close to their activation energy barrier of $5.6 \mathrm{eV}$, as computationally profiled by Ziletti et al. ${ }^{28}$ Therefore, among the significant ambient gases, oxygen has a promising capability of phosphorene degradations under ambient conditions.

Dynamics of Oxygen over Phosphorene. Although DFT reveals that phosphorene can degrade in the presence of oxygen, corresponding dynamics cannot be addressed using the same. Moreover, DFT does not reflect the role of water in the degradation, as observed in the earlier experiments, ${ }^{12,26}$ probably due to the lack of phonon dynamics in the calculations. To probe further, MD of phosphorene degradation was captured until $2000 \mathrm{fs}$ in $1 \mathrm{fs}$ timestamp at $300 \mathrm{~K}$ in the presence of oxygen and water molecules. The dynamics of $\mathrm{O}_{2}$ over pristine phosphorene (Figure 3) confirms the spontaneous dissociation of oxygen over the phosphorene surface. 


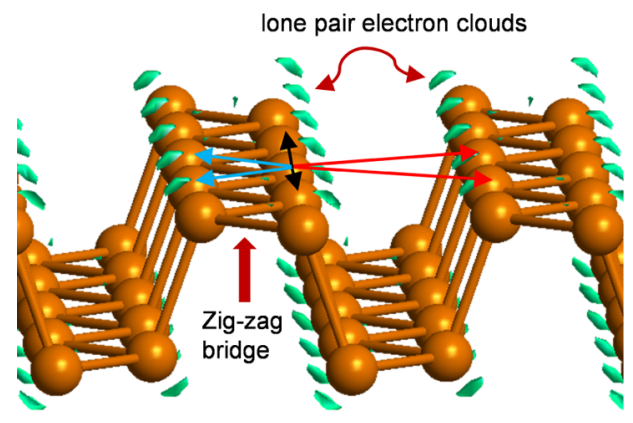

(a)

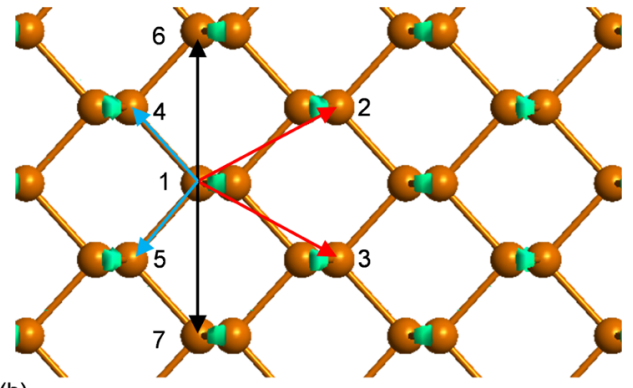

(b)

Figure 6. (a) Tilted front view and (b) top view of a phosphorene layer with lone pair electron clouds (green patches). The isovalue of the electron density surface is $0.78 \AA^{-3}$. All arrows indicate possible approaching oxygen orientations. Same color arrows represent similar neighbor atoms.

The molecule dissociates after 1200 fs (Figure 3d) from the start of the simulation, followed by the dissociated atoms settled on the dangling and bridge positions in the form of surface oxides (Figure 3f). The dangling and average bridge $\mathrm{P}-\mathrm{O}$ bond lengths are 1.53 and $1.71 \AA$, respectively, which are higher than the corresponding DFT results (1.51 and $1.66 \AA$, respectively, as discussed above) due to the incorporation of temperature in the $\mathrm{MD}$ calculation.

The preferred $\mathrm{O}_{2}$ orientation before degradation is parallel to the phosphorene basal plane. $\mathrm{O}_{2}$ aligns from the perpendicular to the parallel position before it dissociates (Figure $3 \mathrm{a}-\mathrm{d}$ ). To confirm this further, the number of oxygen molecules inside the phosphorene supercell (partial pressure of $\mathrm{O}_{2}$ ) was increased with different orientations (Figure 4). The molecules parallel to the phosphorene plane dissociate quickly (Figure 4c), whereas the perpendicular molecules aligned themselves parallel to the plane before breaking off (Figure $4 \mathrm{~d}-\mathrm{e})$. Once oxygen approaches parallel to the phosphorene plane, two $\mathrm{P}-\mathrm{O}$ bonds are formed during the transition phase of the $\mathrm{O}_{2}$ dissociation. The formation of these two bonds provides enough energy to cross the activation barrier to break the oxygen molecule at $300 \mathrm{~K}$. In contrast, one $\mathrm{P}-\mathrm{O}$ bond in the transition phase cannot cross the activation barrier in a similar condition if $\mathrm{O}_{2}$ approaches perpendicular to the phosphorene plane. Thus, the desired $\mathrm{O}_{2}$ orientation is parallel to the phosphorene plane during the degradation process. At high $\mathrm{O}_{2}$ partial pressure, relatively, a higher number of oxygen molecules can align parallel to the phosphorene plane and cause faster degradation. Corresponding degradation kinetics, i.e., energy profiles as a function of time (Figure 5), depicts that reaction with a single $\mathrm{O}_{2}$ molecule has a high activation energy $(\sim 6.4 \mathrm{eV})$ and is endothermic at room temperature (Figure 5). The computed energy profile and activation energy are significantly different from the corresponding static DFT calculation by Ziletti et al. $^{28}$ due to the incorporation of temperature and time evolution parameters. Once oxygen partial pressure is increased (to five $\mathrm{O}_{2}$ molecules), the activation energy is reduced $(\sim 3.7 \mathrm{eV})$ and the process becomes strongly exothermic, which increases the local temperature of phosphorene (around 500 fs timestamp) during the dissociation process (Figure $5 b$ ). Therefore, the phosphorene degradation rate increases once oxygen partial pressure is increased in the system.

Lone Pair-Assisted Directional Etching. Each phosphorous atom in the phosphorene has a lone pair electron oriented outside its basal plane (Figure 6a). Once oxygen approaches, it can interact with the lone pairs and/or $\mathrm{P}-\mathrm{P}$ bonds to initiate the oxidation. The involvement of $\mathrm{P}-\mathrm{P}$ bonds can increase the activation energy because of the bond-breaking phenomenon during transition states, thus slowing down the degradation rate. Therefore, lone pairs are the favorable approaching sites for oxygen. Suppose an oxygen molecule approaches a zigzag bridge's phosphorous atom (atom 1 in Figure 6b), in that case, its other atom can align parallel to the phosphorene surface along any of the nearest atoms of the adjacent zigzag bridge (atoms 2, 3 in Figure 6b), zigzag bonds in the same bridge (atoms 4, 5 in Figure 6b), or nearest atoms in the same zigzag bridge line (atoms 6,7 in Figure 6b). All four directions in the same zigzag bridge (atoms 4, 5, 6, and 7) have higher bonded electron crowding than the other two directions toward the adjacent bridge (atoms 2 and 7 ). The bonded electron crowding can increase the activation energy by repulsion between these crowded electrons with $\mathrm{O}-\mathrm{O}$ bonded electrons. Moreover, electron clouds of the adjacent zigzag bridges face toward each other (Figure 6a), which can bond directly to aligned $\mathrm{O}_{2}$ without any further orientation. Therefore, the preferred $\mathrm{O}-\mathrm{O}$ bond alignment is along the phosphorous atoms of the adjacent zigzag bridge to begin the degradation process (Figures 3 and 4). Hence, the armchair direction of phosphorene can be relatively more prone to surface degradation than the zigzag direction at any given temperature and pressure, which is due to a more significant number of adjacent zigzag bridges. Unlike oxygen molecules, oxygen atoms (free radicals) do not need a proper alignment before phosphorene surface oxidation. It reacts once it comes near the phosphorous lone pair (Supporting Information, Figure S2). Thus, phosphorene can degrade rapidly in the presence of oxygen plasma, which has oxygen radicals too, compared with oxygen molecules under similar system parameters, as also reported by Naclerio et al. ${ }^{25}$

The alignment of $\mathrm{O}_{2}$ parallel to the phosphorene plane hints at a possible protection/passivation strategy. Given that the easy option for $\mathrm{O}_{2}$ to diffuse inside the encapsulated material is perpendicular to the phosphorene plane, to mitigate the diffusion process, phosphorene can be encapsulated by a material whose interatomic gap is less than the $\mathrm{O}_{2}$ bond length. In such a case, it will be tough for $\mathrm{O}_{2}$ to align parallel to the phosphorene plane and cause any degradation unless the encapsulated phosphorene is not exposed to higher temperatures. If such a compact material exists or is synthesized, phosphorene can be ambient stable under passivation of the material.

Enhanced Degradation Catalyzed By Water Molecules. Unlike oxygen, water does not show any affinity toward pristine phosphorene at room temperature. The water molecule tends to stay away from phosphorene during 


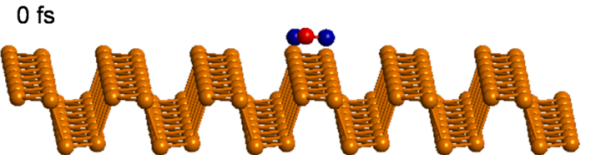

(a)
$2000 \mathrm{fs}$

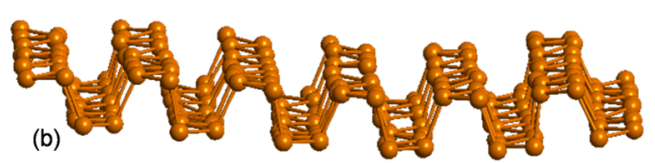

Figure 7. Status of $\mathrm{H}_{2} \mathrm{O}$ molecules over pristine phosphorene at the (a) beginning ( $0 \mathrm{fs}$ ) and (b) end (2000 fs) of $\mathrm{MD}$ simulation at $300 \mathrm{~K}$. $\mathrm{H}_{2} \mathrm{O}$ prefers to stay away from the pristine phosphorene surface. Corresponding full dynamics video is available in the Supporting Information (SV3).

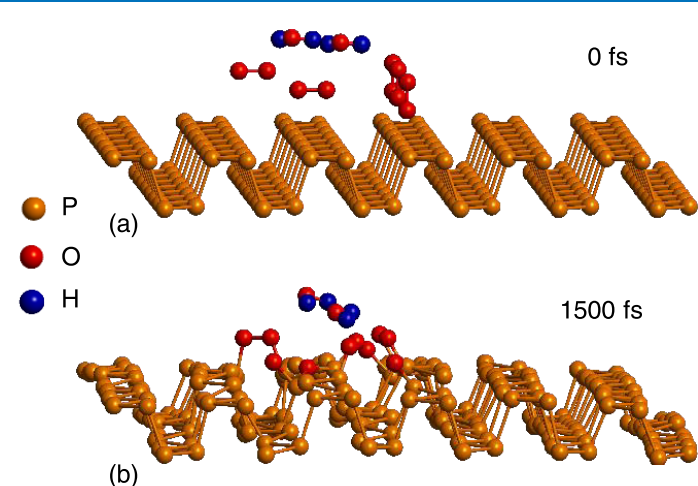

(b)

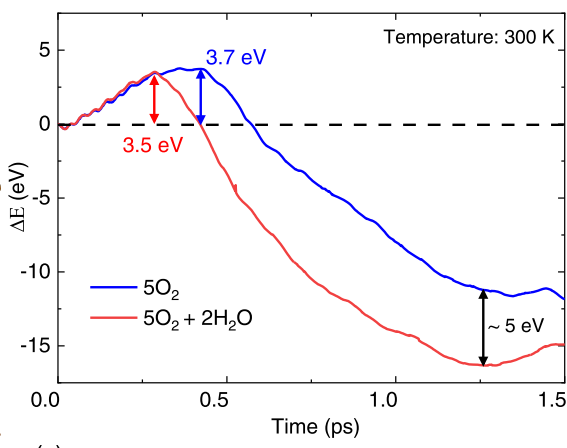

(c)

Figure 8. Status of phosphorene degradation $\mathrm{MD}$ in the presence of five $\mathrm{O}_{2}$ and two $\mathrm{H}_{2} \mathrm{O}$ molecules after (a) 0 fs and (b) $1500 \mathrm{fs}$. (c) $\mathrm{MD}$ energy comparison of the same with phosphorene degradation in the presence of five $\mathrm{O}_{2}$ molecules only (Figure 4). Corresponding full dynamics video is available in the Supporting Information (SV4).

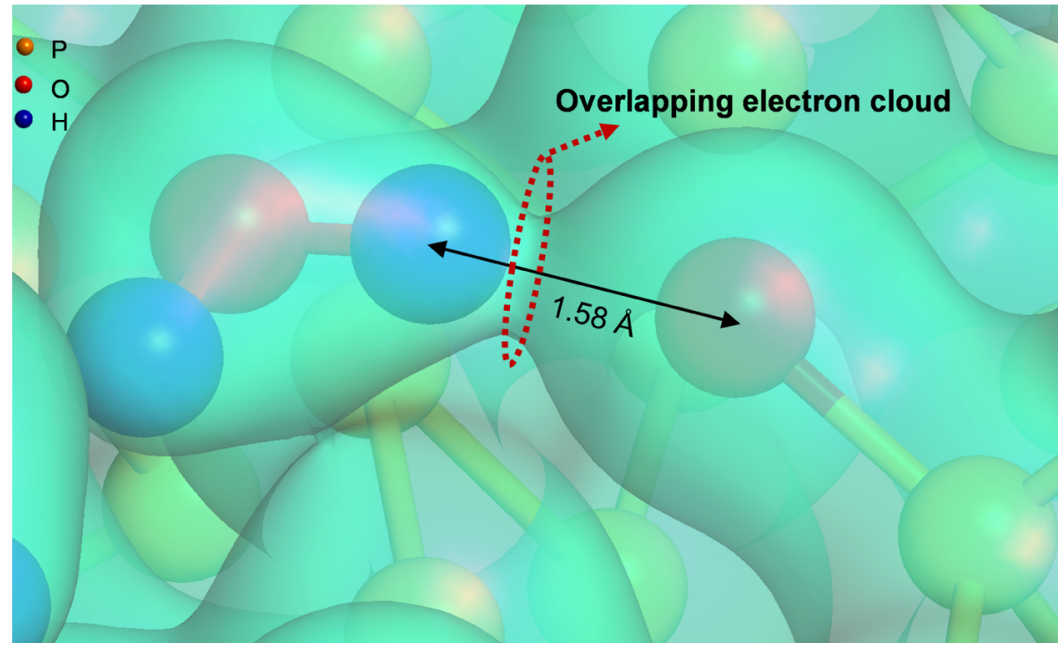

Figure 9. Electron density surface during $\mathrm{MD}$ progress (1653 fs) of phosphorene with five $\mathrm{O}_{2}$ and two $\mathrm{H}_{2} \mathrm{O}$ molecules (Figure 8). The isovalue of the electron cloud surface is $0.3 \AA^{-3}$.

molecular dynamics progress (Figure 7); thus, pristine phosphorene is hydrophobic at room temperature. However, the water molecule influences the degradation kinetics of pristine phosphorene under oxygen by its catalytic action, as observed in the MD study of phosphorene in the presence of oxygen and water molecules (Figure 8 ). Therefore, while water does not involve directly in the covalent bonding process with phosphorous atoms during the degradation process (Figure $8 \mathrm{a}, \mathrm{b})$, it can have a hydrogen bonding interaction with oxygen during the degradation process. The extra energy released from the hydrogen bonding reduces the overall activation energy
$(0.2 \mathrm{eV})$ required for pristine phosphorene degradation, as shown in Figure $8 \mathrm{c} . \mathrm{H}_{2} \mathrm{O}$ not only helps $\mathrm{O}_{2}$ molecules cross the activation energy barrier early in the reaction but also stays near the oxidized surface like strongly adsorbed molecules. It comes so close to the dangling oxygen that the hydrogen and dangling oxygen distance is only $1.58 \AA$ A. In such proximity, the electron cloud of oxygen partially overlaps with the hydrogen electron cloud (Figure 9), which accounts for $\sim 5 \mathrm{eV}$ extra released energy after degradation (Figure 8c). Strong adsorption of water over the oxidized phosphorene surface (partially bonded) reflects that the phosphorene surface has 

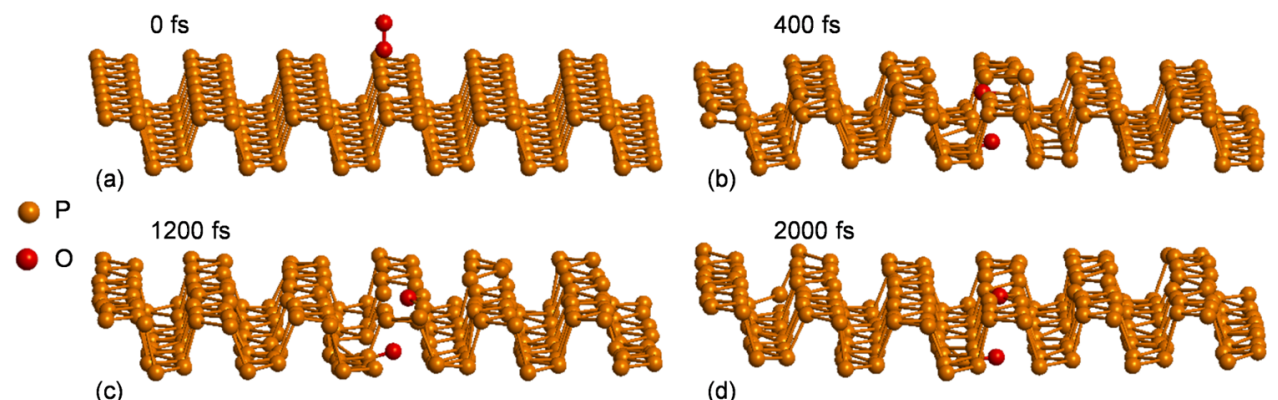

(d)

Figure 10. Status of an $\mathrm{O}_{2}$ molecule over the phosphorous vacancy site of phosphorene in different timestamps, (a) $0 \mathrm{fs}$, (b) $400 \mathrm{fs}$, (c) $1200 \mathrm{fs}$, and (d) $2000 \mathrm{fs}$, during MD progress. Corresponding full dynamics video is available in the Supporting Information (SV5).
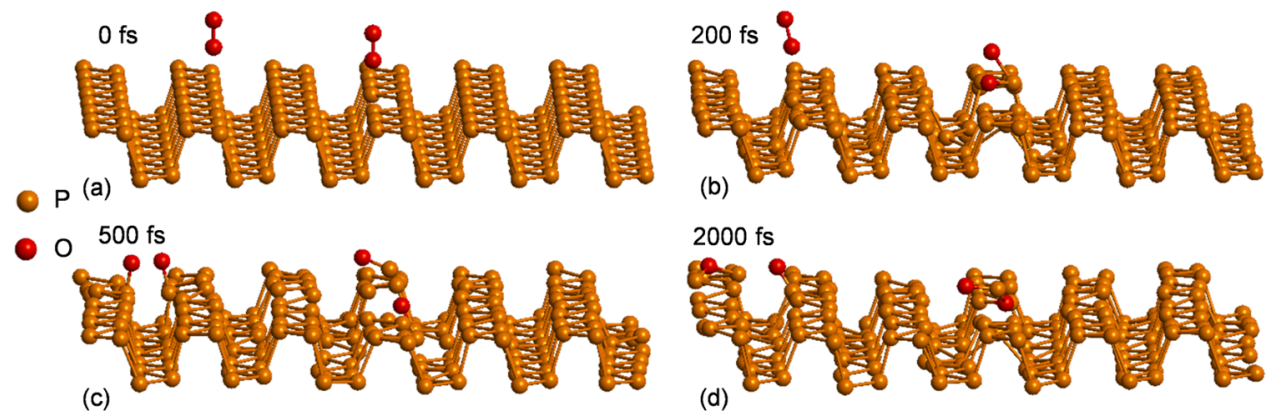

Figure 11. Status of $\mathrm{O}_{2}$ molecules over phosphorous vacancy as well as pristine sites of phosphorene in different timestamps, (a) $0 \mathrm{fs}$, (b) $200 \mathrm{fs}$, (c) $500 \mathrm{fs}$, and (d) $2000 \mathrm{fs}$, during MD progress. Corresponding full dynamics video is available in the Supporting Information (SV6).

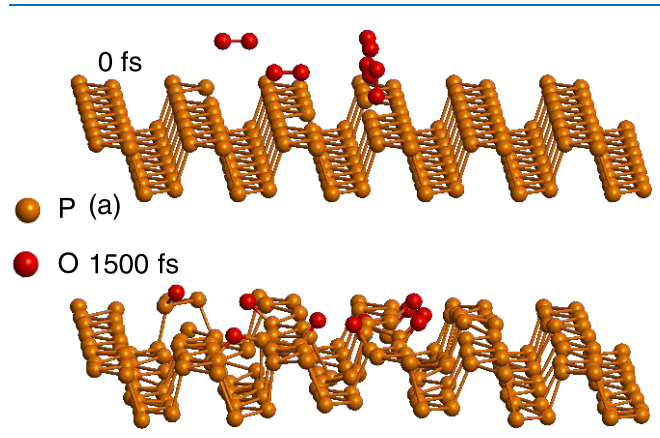

(b)
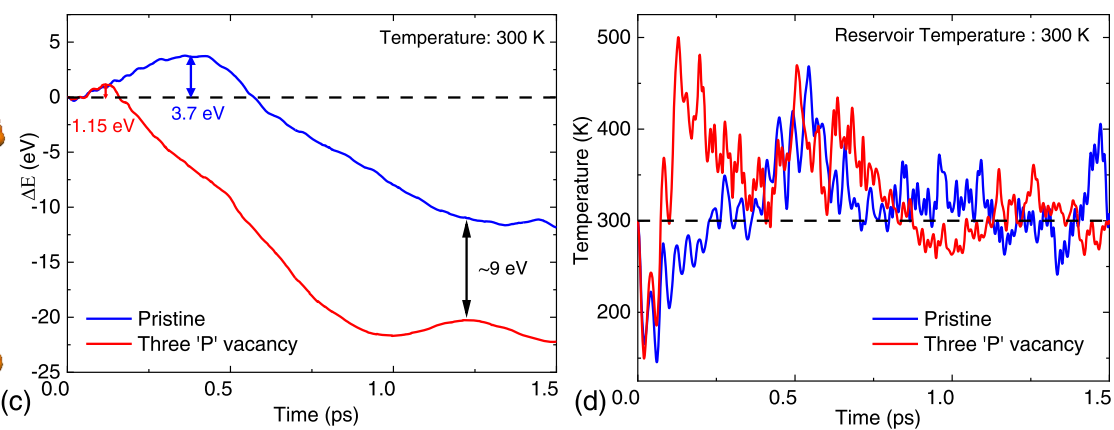

Figure 12. MD status of phosphorene with three phosphorous vacancies in the presence of five $\mathrm{O}_{2}$ after (a) 0 fs and (b) 1500 fs. (c) MD energy and $(\mathrm{d})$ temperature comparisons of the same with pristine phosphorene degradation in the presence of five $\mathrm{O}_{2}$ (Figure 4). Corresponding full dynamics video is available in the Supporting Information (SV7).

been converted into hydrophilic from hydrophobic after surface oxidation. Thus, pristine phosphorene degrades faster in the presence of $\mathrm{O}_{2}$ and $\mathrm{H}_{2} \mathrm{O}$ than $\mathrm{O}_{2}$ alone due to the catalytic action of water and its exothermic interactions with phosphorene surface oxide, as observed earlier in the experimental investigations. ${ }^{12,26}$

Vacancy-Assisted Rapid Degradation. Defects, especially point vacancies, are an integral part of any natural, synthesized, or grown material. Although phosphorous vacancy defect is relatively stable in phosphorene, ${ }^{31}$ it may become critical in the presence of reactive gases, like other twodimensional (2D) materials, ${ }^{32}$ due to unsaturated phosphorous atoms near the vacant sites. To investigate this aspect, $\mathrm{MD}$ studies were performed after adding an oxygen molecule over the vacant site of the phosphorene surface (Figure 10a). Unlike pristine phosphorene, the $\mathrm{O}_{2}$ dissociated near the vacant site quickly (after $400 \mathrm{fs}$ ) and bonded with unsaturated phosphorus atoms (Figure $10 \mathrm{~b}-\mathrm{d}$ ). In the subsequent investigation, once oxygen molecules approach pristine as well as vacant sites together (Figure 11a), it dissociates quickly near the vacant site (after $200 \mathrm{fs}$ ); however, the molecule takes time to align itself before dissociating ( $500 \mathrm{fs}$ ) over the pristine site (Figure $11 \mathrm{~b}-\mathrm{d}$ ). Thus, it can be concluded that phosphorene's surface around the phosphorous vacancy site is prone to degrade faster once exposed to oxygen molecules.

Further, a somewhat generalized case of phosphorene with three vacancy sites under a high partial pressure of oxygen (five $\mathrm{O}_{2}$ molecules) was studied (Figure 12) and compared with a similar study of pristine phosphorene under high pressure. Their reaction energy comparison reflects that due to vacancies, phosphorene degradation is highly exothermic $(\sim 9$ $\mathrm{eV}$ more) with a lower activation barrier (2.55 eV less) than its pristine counterpart (Figure 12c). The rapid exothermic reaction in the presence of vacancy accounts for a quick increase in the system temperature relative to its pristine counterpart (Figure 12d) before releasing the extra energy to 


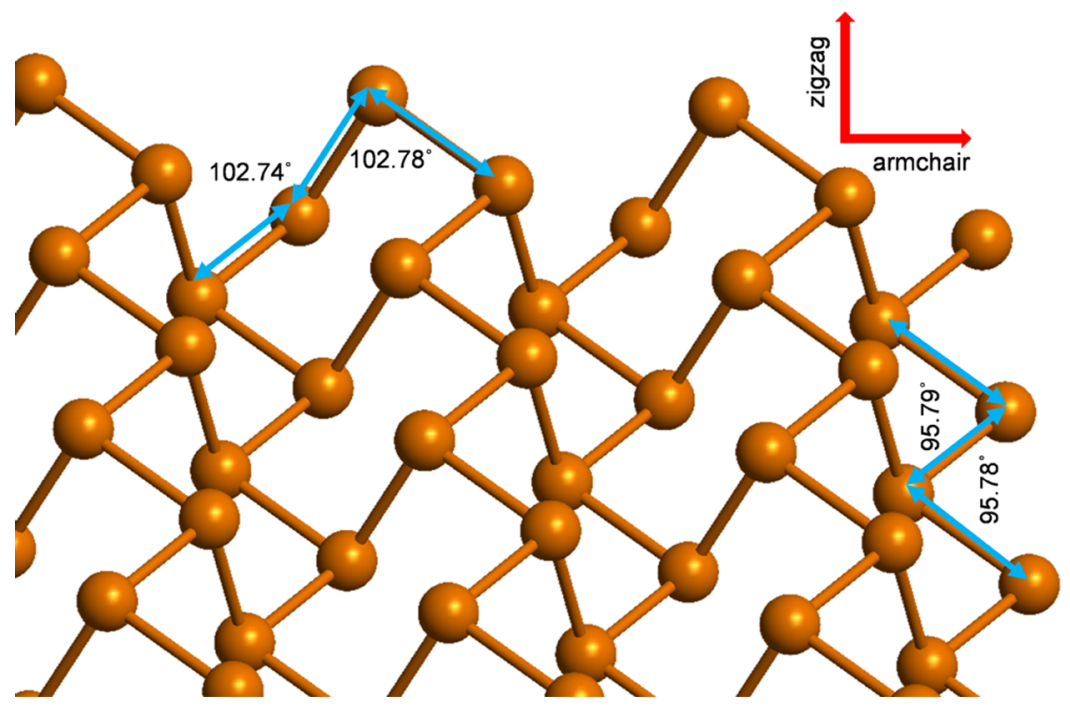

Figure 13. $\mathrm{P}-\mathrm{P}$ bond angles at the armchair and zigzag terminating edges of phosphorene.

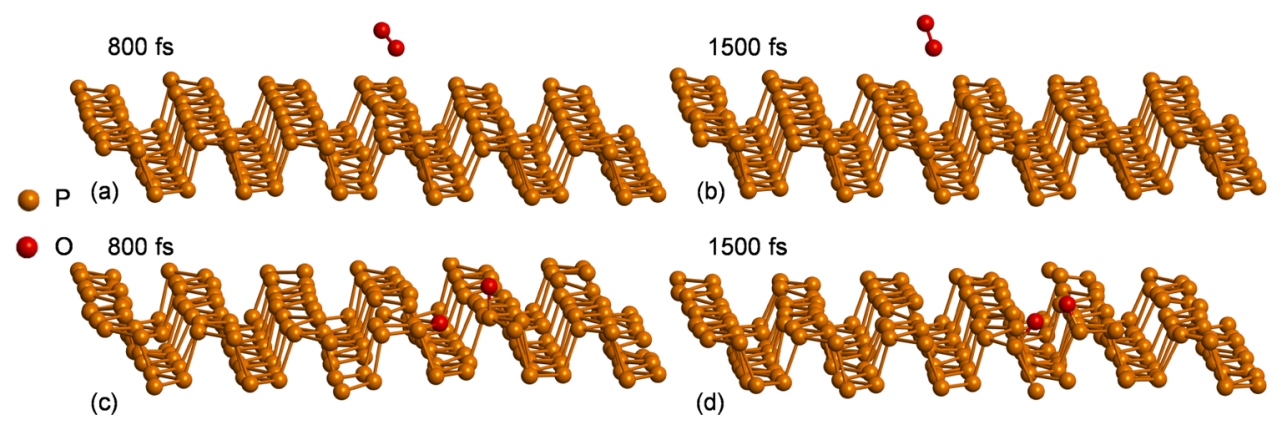

Figure 14. (a, b) Oxygen dissociation status after 800 and $1500 \mathrm{fs}$ in MD investigation at $200 \mathrm{~K}$. (c, d) Oxygen dissociation status after 800 and $1500 \mathrm{fs}$ in MD investigation at $400 \mathrm{~K}$. Corresponding full dynamics videos are available in the Supporting Information (SV8 and SV9, respectively).

the heat reservoir. Therefore, phosphorous vacancy is an absolute killer for phosphorene exposed to oxygen due to unsaturated phosphorous atoms.

Etching Anisotropy at the Edges. While the phosphorene surface is atomically smooth without any dangling bonds, its edges, by default, have unsaturated phosphorous atoms. These unsaturated atoms can act as vacant sites and hence react fast once exposed to oxygen. Given the phosphorene anisotropic structure, its $\mathrm{P}-\mathrm{P}$ bond angles are not the same at the terminating edges. The bond angles are $\sim 102.7$ and $\sim 95.7^{\circ}$ at the zigzag and armchair edges, respectively (Figure 13). Fewer bond angles at the armchair edge account for extra bond repulsion, which renders atoms at the armchair terminating edge relatively more unstable than the zigzag edge. Thus, phosphorene degrades faster at the armchair direction than at the zigzag direction under oxygen (Supporting Information, Figure S3), as also observed by Naclerio et al. ${ }^{25}$ in their experimental investigations. Moreover, the overall degradation rate at the edges is faster than surface degradation. Therefore, edge passivation is highly recommended for the robust application of phosphorene under an ambient environment.

Role of Lattice Temperature. Generally, the rate of any reaction increases with an increase in temperature. Phosphorene degradation under oxygen also follows the same trends, as shown in Figure 14. At $400 \mathrm{~K}$, oxygen dissociates around $800 \mathrm{fs}$ timestamp (Figure 14c,d), while the same does not dissociate until $1500 \mathrm{fs}$ timestamp (Figure 14a,b) at $200 \mathrm{~K}$. The dissociation is faster at a high temperature because an oxygen molecule has higher thermal energy at such high temperatures to overcome activation barrier. In the previous section, we saw that an oxygen molecule over pristine phosphorene at $300 \mathrm{~K}$ dissociates around 1200 fs timestamp in MD investigations (Figure 3). Thus, it can be concluded that the phosphorene degradation rate under oxygen increases with the increase in the system/lattice temperature.

Our investigations provide comprehensive theoretical insights into experimental findings by Huang et al. ${ }^{12}$ They observed that oxygen reacts with BP under ambient conditions. Moreover, pristine BP is hydrophobic, which turns hydrophilic after oxidation. Our investigations are also consistent with the experimental work by Druenen et al. ${ }^{14}$ and theoretical work by Ziletti et al., ${ }^{28}$ who predicted multiple stable positions of oxygen over phosphorene, like dangling, bridge, etc., after degradation. In addition, we have extended and explored further the work done by Wang et al., ${ }^{30}$ who gave an initial glimpse of spontaneous degradation of phosphorene under oxygen using MD calculation. Further, vacancy-assisted fast degradation at the phosphorene edges and its anisotropy explain experimental observations of edge-initiated degradation by Zhang et al. ${ }^{16}$ and anisotropic degradation by Naclerio et al. $^{25}$ Therefore, our first-principles MD investigation provides a systematic theoretical insight into phosphorene degradation under ambient environments. 


\section{CONCLUSIONS}

In summary, we performed systematic investigations of phosphorene degradation under ambient conditions using DFT and the first-principles MD simulations. The roles of oxygen and water molecules, partial pressure, lattice temperature, phosphorous vacancy, and phosphorene's anisotropy were explored. These investigations reveal that oxygen dissociates over phosphorene spontaneously under ambient conditions, which degrades or dissociates phosphorene by oxidizing the surface. Oxygen prefers to align parallel to the phosphorene surface before dissociation because of the formation of two $\mathrm{P}-\mathrm{O}$ bonds, as required during the corresponding transition states. The rate of degradation increases further by increasing the oxygen partial pressure or the lattice temperature. Furthermore, while the pristine phosphorene surface is hydrophobic, it becomes hydrophilic after the surface oxidation, making it attractive to water molecules. Water molecules increase the phosphorene degradation rate by their catalytic action and exothermic interactions with phosphorene surface oxide. Finally, our investigations reveal that phosphorous vacancy acts as an epicenter for phosphorene surface oxidation, responsible for faster degradation around the edges. The edge degradation rate is higher along the armchair direction when compared to the zigzag direction, which is attributed to different atomic stabilities at the edges. These findings can help to engineer passivation schemes for phosphorene devices and make them robust against events responsible for degradation under ambient conditions.

\section{COMPUTATIONAL PARAMETERS}

All of the investigations were done using the QuantumATK computational package. $^{33,34}$ For only $\mathrm{DFT}^{35}$ and firstprinciples $\mathrm{MD}(\mathrm{DFT}+\mathrm{MD})^{30}$ simulations, $5 \times 1 \times 5$ and 8 $\times 1 \times 6$ supercells modules were used, respectively. For DFT, energy optimizations were done with $0.01 \mathrm{eV} / \AA$ force and $0.001 \mathrm{eV} / \AA^{3}$ energy cutoffs, respectively. Perdew-BurkeErnzerhof (PBE) form of the generalized gradient approximation (GGA) ${ }^{36}$ functional was used in the computation with $5 \times 1 \times 5 k$-point sampling (Supporting Information, Figure S1) for all of the modules. Grimme-D2 van der Waals (vdW) correction $^{37}$ was considered in the calculations to capture longrange vdW interactions. Enough vacuum space $(20-30 \AA)$ was added in all of the supercells to avoid interlayer wavefunction interaction during parodic boundary conditions. Hybrid functionals are better than GGA functionals in calculating the structural and fundamental properties of phosphorene at the cost of a high computational budget. ${ }^{38,39}$ However, vdW correction is a significant parameter for better accuracy in computational investigations of adsorption/absorption kinetics. Thus, we used the GGA functional to incorporate the $\mathrm{vdW}$ correction in the calculations under an optimized computational budget.

NPT (constant temperature and pressure) ensemble was used in all of the $\mathrm{MD}$ simulations to replicate general environmental conditions. NPT Martyna Tobias Klein ${ }^{40}$ algorithm was used in the calculations, which had 1 fs calculation timestamp along with 100 and 500 fs of thermostat and barostat timescales, respectively. The DFT method was used to calculate the potential profile in each MD timestamp cycle. Except for different temperature studies, all of the calculations were done at $300 \mathrm{~K}$, where the initial velocity of the atoms follows Maxwell-Boltzmann distribution at the study temperature.

\section{ASSOCIATED CONTENT}

\section{(I) Supporting Information}

The Supporting Information is available free of charge at https://pubs.acs.org/doi/10.1021/acsomega.1c05353.

Plot of the $k$-point convergent test; phosphorene degradation under oxygen atom (free radical); anisotropic edge degradation of phosphorene at different timestamps (PDF); and details of supporting videos

SV1 (MP4)

SV2 (MP4)

SV3 (MP4)

SV4 (MP4)

SV5 (MP4)

SV6 (MP4)

SV7 (MP4)

SV8 (MP4)

SV9 (MP4)

SV10 (MP4)

SV11 (MP4)

\section{AUTHOR INFORMATION}

\section{Corresponding Author}

Mayank Shrivastava - Department of Electronic Systems

Engineering, Indian Institute of Science, Bangalore 560012,

India; (1) orcid.org/0000-0003-1005-040X;

Phone: 09591140309; Email: mayank@iisc.ac.in

Author

Jeevesh Kumar - Department of Electronic Systems

Engineering, Indian Institute of Science, Bangalore 560012, India; $\odot$ orcid.org/0000-0001-6178-8434

Complete contact information is available at:

https://pubs.acs.org/10.1021/acsomega.1c05353

\section{Notes}

The authors declare no competing financial interest.

\section{ACKNOWLEDGMENTS}

The authors thank the NNetRA program of MeitY, DST, and DRDO, as well as CSIR, and MHRD Govt. of India, for supporting this work.

\section{REFERENCES}

(1) Li, L.; Yu, Y.; Ye, G. J.; Ge, Q.; Ou, X.; Wu, H.; Feng, D.; Chen, X. H.; Zhang, Y. Black Phosphorus Field-Effect Transistors. Nat. Nanotechnol. 2014, 9, 372-377.

(2) Das, S.; Demarteau, M.; Roelofs, A. Ambipolar Phosphorene Field Effect Transistor. ACS Nano 2014, 8, 11730-11738.

(3) Liu, H.; Du, Y.; Deng, Y.; Ye, P. D. Semiconducting Black Phosphorus: Synthesis, Transport Properties and Electronic Applications. Chem. Soc. Rev. 2015, 44, 2732-2743.

(4) Kuriakose, S.; Ahmed, T.; Balendhran, S.; Bansal, V.; Sriram, S.; Bhaskaran, M.; Walia, S. Black Phosphorus: Ambient Degradation and Strategies for Protection. 2D Mater. 2018, 5, No. 032001.

(5) van Druenen, M. Degradation of Black Phosphorus and Strategies to Enhance Its Ambient Lifetime. Adv. Mater. Interfaces 2020, 7, No. 2001102.

(6) Zhang, J.; Shin, H.; Lu, W. Highly Ambient-Stable Few-Layer Black Phosphorene by Pulsed Laser Exfoliation and HEMM. Chem. Commun. 2019, 55, 2601-2604. 
(7) Hanlon, D.; Backes, C.; Doherty, E.; Cucinotta, C. S.; Berner, N. C.; Boland, C.; Lee, K.; Harvey, A.; Lynch, P.; Gholamvand, Z.; Zhang, S.; Wang, K.; Moynihan, G.; Pokle, A.; Ramasse, Q. M.; McEvoy, N.; Blau, W. J.; Wang, J.; Abellan, G.; Hauke, F.; Hirsch, A.; Sanvito, S.; O’Regan, D. D.; Duesberg, G. S.; Nicolosi, V.; Coleman, J. N. Liquid Exfoliation of Solvent-Stabilized Few-Layer Black Phosphorus for Applications beyond Electronics. Nat. Commun. 2015, 6, No. 8563

(8) Pei, J.; Gai, X.; Yang, J.; Wang, X.; Yu, Z.; Choi, D. Y.; LutherDavies, B.; Lu, Y. Producing Air-Stable Monolayers of Phosphorene and Their Defect Engineering. Nat. Commun. 2016, 7, No. 10450.

(9) Hyun, C.; Kim, J. H.; Lee, J. Y.; Lee, G. H.; Kim, K. S. Atomic Scale Study of Black Phosphorus Degradation. RSC Adv. 2020, 10, $350-355$.

(10) Wu, S.; He, F.; Xie, G.; Bian, Z.; Luo, J.; Wen, S. Black Phosphorus: Degradation Favors Lubrication. Nano Lett. 2018, 18, 5618-5627.

(11) Kuntz, K. L.; Wells, R. A.; Hu, J.; Yang, T.; Dong, B.; Guo, H.; Woomer, A. H.; Druffel, D. L.; Alabanza, A.; Tománek, D.; Warren, S. C. Control of Surface and Edge Oxidation on Phosphorene. ACS Appl. Mater. Interfaces 2017, 9, 9126-9135.

(12) Huang, Y.; Qiao, J.; He, K.; Bliznakov, S.; Sutter, E.; Chen, X.; Luo, D.; Meng, F.; Su, D.; Decker, J.; Ji, W.; Ruoff, R. S.; Sutter, P. Interaction of Black Phosphorus with Oxygen and Water. Chem. Mater. 2016, 28, 8330-8339.

(13) Island, J. O.; Steele, G. A.; van der Zant, H. S. J.; CastellanosGomez, A. Environmental Instability of Few-Layer Black Phosphorus. $2 D$ Mater. 2015, 2, No. 011002.

(14) van Druenen, M.; Davitt, F.; Collins, T.; Glynn, C.; O’Dwyer, C.; Holmes, J. D.; Collins, G. Evaluating the Surface Chemistry of Black Phosphorus during Ambient Degradation. Langmuir 2019, 35, 2172-2178

(15) Abellán, G.; Wild, S.; Lloret, V.; Scheuschner, N.; Gillen, R.; Mundloch, U.; Maultzsch, J.; Varela, M.; Hauke, F.; Hirsch, A. Fundamental Insights into the Degradation and Stabilization of Thin Layer Black Phosphorus. J. Am. Chem. Soc. 2017, 139, 10432-10440.

(16) Zhang, T.; Wan, Y.; Xie, H.; Mu, Y.; Du, P.; Wang, D.; Wu, X.; Ji, H.; Wan, L. Degradation Chemistry and Stabilization of Exfoliated Few-Layer Black Phosphorus in Water. J. Am. Chem. Soc. 2018, 140, 7561-7567.

(17) Li, W.; Wang, Z.; Zhao, F.; Li, M.; Gao, X.; Zhao, Y.; Wang, J.; Zhou, J.; Hu, Y.; Xiao, Q.; Cui, X.; Eslamibidgoli, M. J.; Eikerling, M. H.; Li, R.; Brandys, F.; Divigalpitiya, R.; Sham, T.-K.; Sun, X. Phosphorene Degradation: Visualization and Quantification of Nanoscale Phase Evolution by Scanning Transmission X-Ray Microscopy. Chem. Mater. 2020, 32, 1272-1280.

(18) Plutnar, J.; Sofer, Z.; Pumera, M. Products of Degradation of Black Phosphorus in Protic Solvents. ACS Nano 2018, 12, 83908396.

(19) Kang, J.; Wells, S. A.; Wood, J. D.; Lee, J. H.; Liu, X.; Ryder, C. R.; Zhu, J.; Guest, J. R.; Husko, C. A.; Hersam, M. C. Stable Aqueous Dispersions of Optically and Electronically Active Phosphorene. Proc. Natl. Acad. Sci. U.S.A. 2016, 113, 11688-11693.

(20) Serrano-Ruiz, M.; Caporali, M.; Ienco, A.; Piazza, V.; Heun, S.; Peruzzini, M. The Role of Water in the Preparation and Stabilization of High-Quality Phosphorene Flakes. Adv. Mater. Interfaces 2016, 3, No. 1500441.

(21) Gómez-Pérez, J.; Barna, B.; Tóth, I. Y.; Kónya, Z.; Kukovecz, Á. Quantitative Tracking of the Oxidation of Black Phosphorus in the Few-Layer Regime. ACS Omega 2018, 3, 12482-12488.

(22) Wang, H.; Yang, X.; Shao, W.; Chen, S.; Xie, J.; Zhang, X.; Wang, J.; Xie, Y. Ultrathin Black Phosphorus Nanosheets for Efficient Singlet Oxygen Generation. J. Am. Chem. Soc. 2015, 137, 1137611382.

(23) Favron, A.; Gaufrès, E.; Fossard, F.; Phaneuf-Laheureux, A. L.; Tang, N. Y. W.; Lévesque, P. L.; Loiseau, A.; Leonelli, R.; Francoeur, S.; Martel, R. Photooxidation and Quantum Confinement Effects in Exfoliated Black Phosphorus. Nat. Mater. 2015, 14, 826-832.
(24) Kumar, J.; Ansh; Yadav, A.; Singh, A.; Naclerio, A.; Zakharov, D.; Kidambi, P.; Shrivastava, M. Physical Insights into Phosphorene Transistor Degradation under Exposure to Atmospheric Conditions and Electrical Stress. IEEE Int. Reliab. Phys. Symp. Proc. 2020, 1-4.

(25) Naclerio, A. E.; Zakharov, D. N.; Kumar, J.; Rogers, B.; Pint, C. L.; Shrivastava, M.; Kidambi, P. R. Visualizing Oxidation Mechanisms in Few-Layered Black Phosphorus via in Situ Transmission Electron Microscopy. ACS Appl. Mater. Interfaces 2020, 12, 15844-15854.

(26) Hu, Z.; Li, Q.; Lei, B.; Zhou, Q.; Xiang, D.; Lyu, Z.; Hu, F.; Wang, J.; Ren, Y.; Guo, R.; Goki, E.; Wang, L.; Han, C.; Wang, J.; Chen, W. Water-Catalyzed Oxidation of Few-Layer Black Phosphorous in a Dark Environment. Angew. Chem., Int. Ed. 2017, 56, 9131-9135.

(27) Zhang, S.; Zhang, X.; Lei, L.; Yu, X. F.; Chen, J.; Ma, C.; Wu, F.; Zhao, Q.; Xing, B. PH-Dependent Degradation of Layered Black Phosphorus: Essential Role of Hydroxide Ions. Angew. Chem., Int. Ed. 2019, 58, 467-471.

(28) Ziletti, A.; Carvalho, A.; Campbell, D. K.; Coker, D. F.; Castro Neto, A. H. Oxygen Defects in Phosphorene. Phys. Rev. Lett. 2015, $114,26-29$.

(29) Eslamibidgoli, M. J.; Eikerling, M. H. Mechanical and Chemical Stability of Monolayer Black Phosphorous Studied by Density Functional Theory Simulations. J. Phys. Chem. C 2018, 122, 22366-22373.

(30) Wang, G.; Slough, W. J.; Pandey, R.; Karna, S. P. Degradation of Phosphorene in Air: Understanding at Atomic Level. 2D Mater. 2016, 3, No. 025011.

(31) Hu, W.; Yang, J. Defects in Phosphorene. J. Phys. Chem. C 2015, 119, 20474-20480.

(32) Kumar, J.; Ansh; Shrivastava, M. Stone-Wales Defect and Vacancy-Assisted Enhanced Atomic Orbital Interactions between Graphene and Ambient Gases: A First-Principles Insight. ACS Omega 2020, 5, 31281-31288.

(33) Soler, J. M.; Artacho, E.; Gale, J. D.; García, A.; Junquera, J.; Ordejón, P.; Sánchez-Portal, D. The SIESTA Method for Ab Initio Order-N Materials Simulation. J. Phys.: Condens. Matter 2002, 14, $2745-2779$.

(34) QuantumATK R-2020.09.

(35) Kohn, W.; Sham, L. J. Self-Consistent Equations Including Exchange and Correlation Effects. Phys. Rev. 1965, 140, A1133.

(36) Perdew, J. P.; Burke, K.; Ernzerhof, M. Generalized Gradient Approximation Made Simple. Phys. Rev. Lett. 1996, 77, 3865-3868.

(37) Grimme, S. Semiempirical GGA-Type Density Functional Constructed with a Long-Range Dispersion Correction. J. Comput. Chem. 2006, 27, 1787-1799.

(38) Çaklr, D.; Sevik, C.; Peeters, F. M. Significant Effect of Stacking on the Electronic and Optical Properties of Few-Layer Black Phosphorus. Phys. Rev. B 2015, 92, No. 165406.

(39) Allec, S. I.; Wong, B. M. Inconsistencies in the Electronic Properties of Phosphorene Nanotubes: New Insights from LargeScale DFT Calculations. J. Phys. Chem. Lett. 2016, 7, 4340-4345.

(40) Martyna, G. J.; Tobias, D. J.; Klein, M. L. Constant Pressure Molecular Dynamics Algorithms. J. Chem. Phys. 1994, 101, 41774189. 